\title{
BMJ Open Assessing the impact of care pathways on potentially preventable complications and costs for spinal trauma patients: protocol for a data linkage study using cohort study and administrative data
}

Bharat Phani Vaikuntam, ${ }^{1,2}$ James W Middleton, ${ }^{1,2,3}$ Patrick McElduff, ${ }^{4}$ Jim Pearse,${ }^{4}$ John Walsh, ${ }^{1,2}$ Ian D Cameron, ${ }^{2}$ Lisa Nicole Sharwood ${ }^{1,2}$

To cite: Vaikuntam BP, Middleton JW, McElduff $P$, et al. Assessing the impact of care pathways on potentially preventable complications and costs for spinal trauma patients: protocol for a data linkage study using cohort study and administrative data. BMJ Open 2018;8:e023785. doi:10.1136/ bmjopen-2018-023785

- Prepublication history and additional material for this paper are available online. To view these files, please visit the journal online (http://dx.doi. org/10.1136/bmjopen-2018023785).

Received 27 April 2018 Revised 12 September 2018 Accepted 26 September 2018

Check for updates

(C) Author(s) (or their employer(s)) 2018. Re-use permitted under CC BY-NC. No commercial re-use. See rights and permissions. Published by BMJ.

For numbered affiliations see end of article.

Correspondence to Bharat Phani Vaikuntam; bvai6198@uni.sydney.edu.au

\section{ABSTRACT}

Introduction Traumatic spinal cord injuries have significant consequences both for the injured individual and the healthcare system, usually resulting in lifelong disability. Evidence has shown that timely medical and surgical interventions can lead to better patient outcomes with implicit cost savings. Potentially preventable secondary complications are therefore indicators of the effectiveness of acute care following traumatic injury. The extent to which policy and clinical variation within the healthcare service impact on outcomes and acute care costs for patients with traumatic spinal cord injury (TSCl) in Australia is not well described.

Methods and analysis A comprehensive data set will be formed using record linkage to combine patient health and administrative records from seven minimum data collections (including costs), with an existing data set of patients with acute TSCI (Access to Care Study), for the time period June 2013 to June 2016. This person-level data set will be analysed to estimate the acute care treatment costs of TSCI in New South Wales, extrapolated nationally. Subgroup analyses will describe the associated costs of secondary complications and regression analysis will identify drivers of higher treatment costs. Mapping patient care and health service pathways of these patients will enable measurement of deviations from best practice care standards and cost-effectiveness analyses of the different pathways.

Ethics and dissemination Ethics approval has been obtained from the New South Wales Population and Health Services Research Ethics Committee. Dissemination strategies include peer-reviewed publications in scientific journals and conference presentations to enable translation of study findings to clinical and policy audiences.

\section{INTRODUCTION}

Traumatic spinal cord injury (TSCI) is a devastating injury, most often being life-altering and causing lifelong disability. The ongoing
Strengths and limitations of this study

- The effective use of the Centre for Health Record's capacity to link to additional data sets that are not routinely part of the administrative data collections enables a richer subset of data within which to examine the costs and care provided for patients with traumatic spinal cord injury (TSCl) in the state of New South Wales, Australia.

The use of New South Wales Activity Based Funding District and Network Return Data is a novel strategy to capture the true costs of treatment for patients with TSCI in acute care settings at the hospital level.

- The costs are limited to the perspective of the healthcare provider with direct social care costs and indirect costs from loss of productivity to patients or caregivers not included, and thereby underestimate the financial impact of TSCl to the society as a whole.

physical, mental and economic consequences of this type of injury have substantial effects on patients and their families. The equally enduring economic responsibilities on the healthcare service fall similarly on personal health insurance, as well as disability and injury insurance providers. The cost of spinal cord injury in Australia was last estimated at $\$ 2$ billion annually in $2008^{1}$; consumer price indexation adjusts this to $\$ 2.38$ billion in 2017. ${ }^{2}$ The majority $(79 \%)$ of all new spinal cord injury cases reported to specialist spinal cord injury units (SCIU) are attributable to trauma and as such, the burden of TSCI on the Australian healthcare system is high. ${ }^{3}$ Improvements in prehospital and early trauma care have seen improved survival rates after severe traumatic injury, ${ }^{4}$ shifting 
a greater morbidity burden to lifetime injury. As this continues, the long-term costs of ongoing disability associated with severe injury will also rise.

The incidence of TSCI in Australia is reported between 15.0 and 32.3 cases per million population per year, with an estimated prevalence of 10000-20 000 Australians living with an SCI. ${ }^{5-7}$ Very high treatment costs for patients with TSCI in an acute care setting have been described in the international context ${ }^{8}$; however, updated evaluation of treatment costs for patients with TSCI in Australia is needed. It is vital to contextualise these costs in the present day configuration of acute care settings and specialist services. This picture must be completed with exploration and clarification of the drivers of higher treatment costs, and in particular, any measurable association with clinical variation in the care of patients with TSCI. The prevalence and cost of potentially preventable complications for patients with TSCI need exploration in the Australian healthcare setting in order to drive health service improvement nationally.

Evidence has shown that failure to provide care according to specific guidelines in a timely manner for the patient with TSCI can directly affect both patient outcomes and the costs of rehabilitation and treatment. ${ }^{10} 11$ During the immediate postinjury phase, patients with TSCI are at high risk of secondary neurological damage related to neural tissue oedema, increased cord compression, altered blood flow and reduced tissue perfusion, which can lead to permanent neurological degeneration. Timely medical and surgical interventions can prevent or reduce the extent of secondary neurological injury and lead to better patient outcomes with implicit cost savings. ${ }^{12}{ }^{13}$ It is further well described that specific acute care protocols must be followed to avoid preventable secondary complications such as pressure injuries, urinary tract or respiratory infections, which may otherwise severely hamper long-term recovery and outcomes.

Significant variations in policy and practice may exist among healthcare providers based on differences in perceptions of precisely what constitutes 'best-practice' specialist care, including referral patterns to specialist centres and the timing of surgical intervention. ${ }^{14}$ However, there is little evidence on the extent to which variations in clinical practice and institutional performance in Australia affect patient outcomes and acute care costs, and further are amenable to change. This has been identified as a priority by the Australian Institute for Health and Welfare. ${ }^{15}$

This study will examine a data linkage cohort of patients aged 16 years or older, who sustained acute TSCI in the Australian state of New South Wales (NSW) between June 2013 and June 2016 with the following primary aims:

1. To accurately quantify the acute care costs for patients with TSCI across NSW, and explore health service pathway and clinical practice variations potentially attributable to higher cost hospitalisations.
2. To describe the incidence of potentially preventable complications in patients with acute TSCI and the associated incremental healthcare costs.

3. To benchmark actual patient pathways and care against the best practice standards previously defined using a modified e-Delphi process and other sources, quantifying financial costs associated with deviation of care from these standards.

4. To estimate and compare the cost-effectiveness (CE) of treatment pathway options, and construct an efficiency measure for comparison.

Understanding the costs and health service drivers associated with potentially preventable complications should clarify the investment potential in the construction and implementation of a cost-effective 'best-practice' clinical pathway for patients with TSCI. Communication of these findings will therefore aim to drive change on a national scale.

\section{METHODS AND ANALYSIS}

The Access to Care Study ${ }^{7}$ is a cohort study of 202 patients with TSCI, documenting early care protocols and transport decisions from the scene of injury to definitive diagnosis and specialised treatment. Recruitment occurred from June 2013 to January 2016. The NSW Admitted Patient Data Collection (APDC) will be used to identify and extract TSCI patient records from all separations from NSW public hospitals, based on specific TSCI-related International Classification of Diseases and Related Health Problems, 10th version, Australian Modification (ICD-10-AM) codes (online supplementary appendix 1) in their diagnosis codes. Data linkage will be undertaken by the NSW Centre for Health Record Linkage (CHeReL), linking the Access to Care Study data set with the APDC.

The NSW APDC contains demographic and hospitalisation data for every inpatient admission to any public hospital in NSW. These records will be then linked by the CHeReL, with the following administrative patient data sets using a combination of deterministic and probabilistic methods:

- NSW Emergency Department Data Collection (EDDC) which contains data on presentations to emergency departments in most public hospitals in NSW.

- NSW Ambulance Data Collection which contains information from the computer-aided dispatch system and patient health records.

- NSW Registry of Births, Deaths and Marriages Data which records information for deaths occurring in NSW; and the NSW Cause of Death files.

- NSW Activity Based Funding District and Network Return (NSW-DNR) Data Collection on individual patient cost and health service activity data to ensure all cases were included prior to case identification.

The CHeReL uses probabilistic record linkage techniques to link the patient identifiers from the APDC to the other data sets using a best practice protocol for 
preserving the patient privacy. Linked patient healthcare records will include prehospital and acute care administrative data collections and death records, as well as cost data collections to provide a comprehensive data set. The final record linked data set will be analysed to identify the first hospital admission for a patient satisfying the criteria for a TSCI (hereafter called the index admission), providing details of comorbidities, inpatient complications and unplanned readmissions within the study period to address the study objectives. The index admission will be considered the first to have one of the identified ICD-10-AM codes for TSCI in an acute separation (see online supplementary appendix 1); such admissions will most likely have an immediately preceding NSW Ambulance retrieval and transfer record.

\section{Analysis plan}

The study aims are expanded in the following paragraphs.

1. To accurately quantify the acute care costs for patients with TSCI across NSW, and explore health service pathway and clinical practice variations potentially attributable to higher cost hospitalisations.

Data on resource use will be collected using the NSW-DNR data and Australian Refined Diagnostic Related Group (AR-DRG) codes recorded in the APDC and EDDC for each episode of care for patients with TSCI. Subcategorisations within the NSW-DNR include employee-related costs, costs of visiting medical officers, other operating expenses, professional indemnity charges, hospital finance costs, depreciation and amortisation, payments to affiliated health organisations and grant and subsidy costs.

A generalised linear model regression with a log link and gamma error term will be performed on the costs to determine independent predictors of acute care costs. Variables for the severity of the injury such as the ICD 10th revision-based Injury Severity Scores, Charlson Comorbidity Index and the level of injury (cervical, thoracic or lumbar regions) will be included in the model to adjust for the heterogeneity between the patients. Sensitivity analysis will be used to account for the excess costs incurred by comorbidities not attributable to TSCI; decreasing costs by percentage figure relative to the presence of comorbidities at the time of the TSCI. Variation in activity-based costs will be explored to highlight areas of resource use that require further investigation, similar to research conducted recently across the NSW Trauma Service, using this approach. ${ }^{16}$

2. To describe the incidence of potentially preventable complications in patients with acute TSCI and the associated incremental healthcare costs.

Potentially preventable complications (such as pressure ulcers or urinary tract infections) related to the TSCI and within the index admission will be identified within the ICD-10-AM codes, predefined according to published data $^{17}$ and listed in online supplementary appendix 2. Subsequent readmissions from the index admission and within the study period will be scrutinised to determine any ongoing injury sequelae including potentially preventable complications. Readmissions within 30 days from first acute discharge will be principally identified as unplanned readmissions. The cost of these secondary complications will be estimated using the NSW-DNR data and AR-DRG codes recorded for each episode of care.

3. To benchmark actual patient pathways and care against the best practice standards previously defined through a modified e-Delphi process and other sources, quantifying financial costs associated with deviation of care from these standards.

The patient clinical care pathway will be mapped from the scene of injury to definitive care, incorporating numbers of hospital transfers, hospital-specific factors describing healthcare utilisation, length of stay, hospital level and their location. This mapping will be compared with the best practice care standards defined by the modified e-Delphi process ${ }^{18}$ and current state-based guidelines for the management of major trauma to determine the clinical pathway components reflecting optimal timing and care. This comparison will permit factors potentially associated with unwarranted clinical variation for patients with TSCI to be identified.

A gap driver analysis will aim to identify drivers of cost and clinical pathway differences and will consider factors including delayed and/or multiple transfers, delays in timing of surgery, lack of admission to specialist TSCI services and the occurrence of secondary complications. A range of controllable and uncontrollable factors will be measured against the best practice standards. Multilevel regression analysis will be performed to determine patient-level and hospital-level factors associated with cost variability.

4. To estimate and compare the CE of treatment pathway options, and propose a cost-effective clinical pathway.

Decision analytic modelling will be used to build a cost-effective clinical pathway closely emulating the best practice standards. A decision tree will be modelled based on the observed hospital costs, admissions, readmissions, mortality and complications data from the linked health records, Australian life tables, previously mapped care pathways, incidence rates and previously published literature. The total resource use calculated will include the costs for admissions, complications, emergency department visits, surgery, readmissions and diagnostic services, and will be estimated for the analysis and presented in Australian dollars. Relevant internal validity measures will be undertaken to affirm the validity of the model. ${ }^{19} \mathrm{~A}$ CE analysis will be conducted to compare the proposed/modelled care pathway against the current care practices identified in the record linked data, from the perspective of the healthcare provider. The model will use the study population and the study settings described above over a period of 12 months after injury for the evaluation. Service utilisation data attached to each unique health record will be extracted and analysed based on the relevance of AR-DRG codes to the ICD-10 codes of interest. Cost weights associated with each AR-DRG will be adjusted for differences for each financial year during 
the entire study period. All cost data will be inflated or deflated as required using the published health deflators and a discount rate of $5 \%$ will be used in the evaluation. The quality-adjusted life years for each treatment arm for the modelling will be derived from follow-up interview self-reports from the patients in the nested Access to Care Study, combined with estimates from similarly published literature.

These utility measures will be subject to probabilistic sensitivity analysis along with other key parameters in the model over a wide range based on clinical judgement and existing literature. The incremental cost-effectiveness ratios (ICER) will be estimated to compare the interventions using the expected costs and effects from the decision analytic modelling exercise. A CE plane will be used to plot the ICER and the uncertainty will be represented by confidence ellipses, which are a two-dimensional representation of the CIs. ${ }^{20}$

All the data analyses will be conducted using Stata software (V.15.0, STATA, College Station, TX, USA) and the decision analytic modelling will be conducted using the TreeAge Pro software (2017, V.R2).

\section{Patient and public involvement}

The study will use deidentified record linked health data and will not involve any patients or public.

\section{ETHICS AND DISSEMINATION}

Ethics approval has been obtained from the NSW Population and Health Services Research Ethics Committee. The Access to Care Study database contains 202 patients who have consented to access to their medical records; this data set was identified for the purpose of record linkage and returned deidentified. Dissemination of research findings to key stakeholders is envisaged through peer-reviewed publications in scientific journals, conference proceedings and collaborator meetings. The study is ideally placed to establish standards, and reduce costs with potential for research translation across the state of NSW.

\section{DISCUSSION}

The long-term impact of TSCI on our healthcare system is significant and ongoing. Deficits in clinical policy and practice can significantly impact the extent of long-term disability and resultant quality of life for patients with TSCI, thereby escalating personal and healthcare system economic costs. Identification of specific deficits in system performance will facilitate application of targeted improvement strategies, aiming for greater efficiency of our services, focused resource use, highest standard of practice and consequently optimal outcomes. This study is ideally placed to establish standards, reduce costs and translate evidence back to the bedside.

A particular challenge envisaged in this study is the mapping of patient pathways, which may reveal many routes with multiple interactions within the healthcare system. Many individual routes can be defined based on the initial diagnosis, geographical location and the transition pathway. However, to be useful for the analysis, these pathways will need to be aggregated into a manageable number of broader pathways guided primarily by existing policy directives to prehospital and acute care services. Particular limitations are envisaged with the study design; first, the costs are limited to the acute care episode, and direct social care costs or indirect costs from loss of productivity to patients or caregivers are not included. This therefore underestimates the financial impact of TSCI to society as a whole. Despite all the efforts to accurately describe all cost elements of acute care, the possibility of some resources not being captured cannot be excluded. However, we anticipate this aspect at least to be uniform across all subgroups or pathways and not significantly affect the research findings as most of the resources will be accounted for in the costing exercise. Patients with major trauma will have other immediate healthcare needs in addition to spinal cord injury management and may follow a pathway best suited to patient needs rather than the best practice pathway. We anticipate adjusting for some of this variation by conducting a sensitivity analysis with a group of patients with an isolated spinal cord injury and including measures for patient injury severity, comorbidity and multiple trauma in the analyses.

This study proposes to describe the costs of acute care, including those identified as attributable to potentially preventable complications. Care pathway deviations from best practice will be described, such as delayed surgery or delayed admission to SCIU for patients with TSCI. Importantly, we aim to quantify the degree to which variation in clinical practice and institutional performance impacts on patient outcomes and acute care costs and is amenable to change. These findings are likely to have significant clinical consequences for the patient, cost implications for the health service ${ }^{21}$ and offer significant potential to make amenable recommendations for improvement.

\section{Author affiliations}

${ }^{1}$ Sydney Medical School - Northern, University of Sydney, Sydney, New South Wales, Australia

${ }^{2}$ Kolling Institute, John Walsh Centre for Rehabilitation Research, Sydney, New South Wales, Australia

${ }^{3}$ Agency for Clinical Innovation, NSW Statewide Spinal Cord Injury Service, Sydney, New South Wales, Australia

${ }^{4}$ Health Policy Analysis, Sydney, New South Wales, Australia

Acknowledgements We acknowledge the contributions of Professor Luke Connelly to the review of this manuscript.

Contributors LNS is the principal investigator for this funded study and was principally responsible for the conception and design of this study and the acquisition of the record linkage data. JWM, PM, JP, IDC and JW provided substantial intellectual content during the study design. JWM is the principal investigator for the Access to Care Study. LNS is the principal investigator for the Delphi process project. BPV wrote the first draft of the manuscript and coordinated commentary and review for subsequent revisions. All authors contributed to the critical review and approval of the final manuscript.

Funding This project is funded by grants from the Ramsay Research and Teaching Fund and icare. LNS is funded by icare and the John Walsh Centre for Rehabilitation 
Research. This study forms part of a research higher degree project of the primary author supported by icare and The University of Sydney.

Competing interests None declared.

Patient consent Not required.

Ethics approval Ethics approval for this study has been granted by the NSW Population and Health Services Research Ethics Committee on 17 August 2016 (AU RED REF: HREC/16/CIPHS/19)

Provenance and peer review Not commissioned; externally peer reviewed.

Open access This is an open access article distributed in accordance with the Creative Commons Attribution Non Commercial (CC BY-NC 4.0) license, which permits others to distribute, remix, adapt, build upon this work non-commercially, and license their derivative works on different terms, provided the original work is properly cited, appropriate credit is given, any changes made indicated, and the use is non-commercial. See: http://creativecommons.org/licenses/by-nc/4.0/.

\section{REFERENCES}

1. Access Economics. The economic cost of spinal cord injury and traumatic brain injury in Australia. Access Economics for the Victorian Neurotrauma Initiative. Canberra, 2009.

2. Australian Bureau of Statistics. Consumer Price Index. Canberra: Australian Bureau of Statistics, 2017.

3. Norton L. Spinal Cord Injury, Australia, 2007-08. Canberra: Australian Institute of Health and Welfare, 2010.

4. Minei JP, Fabian TC, Guffey DM, et al. Increased trauma center volume is associated with improved survival after severe injury: results of a Resuscitation Outcomes Consortium study. Ann Surg 2014;260:456-65.

5. New PW, Baxter D, Farry A, et al. Estimating the incidence and prevalence of traumatic spinal cord injury in Australia. Arch Phys Med Rehabil 2015;96:76-83.

6. Spinal Cord Injuries Australia. SCI Statistics. NSW: SCIA, 2017.

7. Middleton JM, Sharwood LN, Cameron P, et al. Right care, right time, right place: improving outcomes for people with spinal cord injury through early access to intervention and improved access to specialised care: study protocol. BMC Health Serv Res 2014;14:600.
8. Munce SE, Wodchis WP, Guilcher SJ, et al. Direct costs of adult traumatic spinal cord injury in Ontario. Spinal Cord 2013:51:64-

9. DeVivo MJ. Causes and costs of spinal cord injury in the United States. Spinal Cord 1997:35:809-13.

10. Middleton PM, Davies SR, Anand S, et al. The pre-hospital epidemiology and management of spinal cord injuries in New South Wales: 2004-2008. Injury 2012;43:480-5.

11. Mac-Thiong JM, Feldman DE, Thompson C, et al. Does timing of surgery affect hospitalization costs and length of stay for acute care following a traumatic spinal cord injury? J Neurotrauma 2012;29:2816-22.

12. Rackauskas Z, Lapteva O, Rocka S. Timing of Surgery for Neurologically Compromised Traumatic Cervical Spine Injuries. J Neurol Surg A Cent Eur Neurosurg 2016;77:427-31.

13. Fehlings MG, Vaccaro A, Wilson JR, et al. Early versus delayed decompression for traumatic cervical spinal cord injury: results of the Surgical Timing in Acute Spinal Cord Injury Study (STASCIS). PLOS One 2012; 7:e32037.

14. Graves N, Zheng H. Modelling the direct health care costs of chronic wounds in Australia. Wound Practice \& Research: Journal of the Australian Wound Management Association 2014;22:20.

15. Australian Government. Biennial health report of the Australian Institute of Health and Welfare. Canberra: Australia's Health, 2014.

16. Curtis K, Lam M, Mitchell R, et al. Major trauma: the unseen financial burden to trauma centres, a descriptive multicentre analysis. Aust Health Rev 2014;38:30-7.

17. Middleton JW, Lim K, Taylor L, et al. Patterns of morbidity and rehospitalisation following spinal cord injury. Spinal Cord 2004;42:359-67.

18. Sharwood LN, Stanford R, Middleton JW, et al. Improving care standards for patients with spinal trauma combining a modified e-Delphi process and stakeholder interviews: a study protocol. BMJ Open 2017;7:e012377.

19. McCabe C, Dixon S. Testing the validity of cost-effectiveness models. Pharmacoeconomics 2000:17:501-13.

20. Petrou S, Gray A. Economic evaluation alongside randomised controlled trials: design, conduct, analysis, and reporting. $B M J$ 2011;342:d1548

21. Grimshaw JM, Eccles MP, Lavis JN, et al. Knowledge translation of research findings. Implement Sci 2012;7:50. 